\title{
Transcavernous Approach to the Anteromedial Triangle for Residual Functional Pituitary Adenoma
}

\author{
Daniele Starnoni ${ }^{1}$ Giulia Cossu ${ }^{1}$ Mahmoud Messerer ${ }^{1,2}$ Roy Thomas Daniel ${ }^{1,2}$ \\ ${ }^{1}$ Department of Neurosurgery, Centre Hospitalier Universitaire \\ Vaudois, Switzerland \\ 2 University of Lausanne (UniL), Lausanne, Switzerland

\begin{abstract}
Address for correspondence Roy Thomas Daniel, MCh, Department of Neurosurgery, Centre Hospitalier Universitaire Vaudois, BH 08635 , Rue du Bugnon 46, 1011 Lausanne, Switzerland (e-mail: roy.daniel@chuv.ch).
\end{abstract}

J Neurol Surg B Skull Base 2022;83(suppl S3):e630-e631.

\begin{abstract}
Surgical treatment of functional pituitary adenomas is as rule performed by transsphenoidal approach. However, when then lesion invades the parasellar structures and the cavernous sinus, the transsphenoidal removal of these adenomas is usually incomplete. In this video, we present the technical nuances of a transcavernous approach to the anteriomedial triangle for the resection of a residual functional pituitary adenoma. The patient is a 40-year-old male who was diagnosed with growth hormone secreting pituitary macroadenoma. He underwent two transsphenoidal resections in 2013 and 2016 with a small residue in the left cavernous sinus. Subsequently, due to a failure of biochemical remission despite medical management, a transcranial transcavernous surgery was performed. Brain magnetic resonance imaging showed a mass in the roof of the left cavernous sinus, located at the level of the anteromedial triangle, adherent to the clinoidal segment of the internal carotid artery (ICA). The computed tomographic scan showed an osteolysis of the inferior surface of the anterior clinoidal process. After performing an extended pterional craniotomy and an extradural clinoidectomy, the cleavage plane is extended between the temporal dura and the inner layer of the lateral wall of the cavernous sinus. Intraoperative Doppler

\section{Keywords}

- cavernous sinus

- extradural clinoidectomy

- anteromedial triangle

- pituitary adenoma and stimulation are used to localize the clinoidal segment of the ICA and the third cranial nerve, delimiting the anteromedial triangle. The lesion is progressively dissected and removed. An optic neuropexy with the previously harvested fat is performed in case of a complementary radio surgical treatment. The patient had an uneventful postoperative course and showed a biochemical remission at the 3-month follow-up.

The link to the video can be found at: https://youtu.be/oHfugVtU-Nc.
\end{abstract}

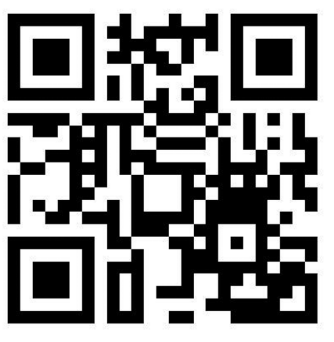

received

April 1, 2020

accepted

November 3, 2020

published online

May 11, 2021

www.thieme.com/skullbasevideos

www.thieme.com/jnlsbvideos

DOI https://doi.org/ $10.1055 / \mathrm{s}-0041-1727119$ ISSN 2193-6331.

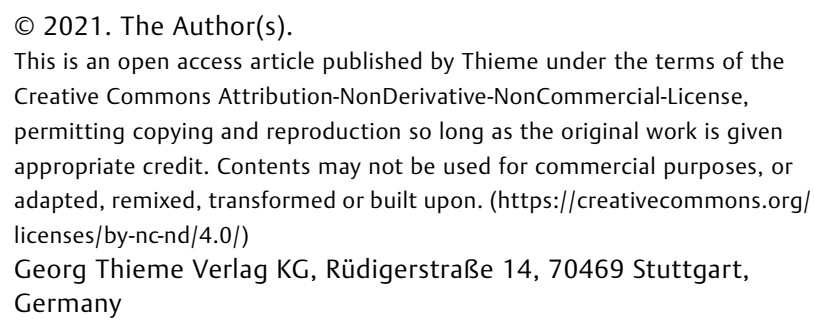




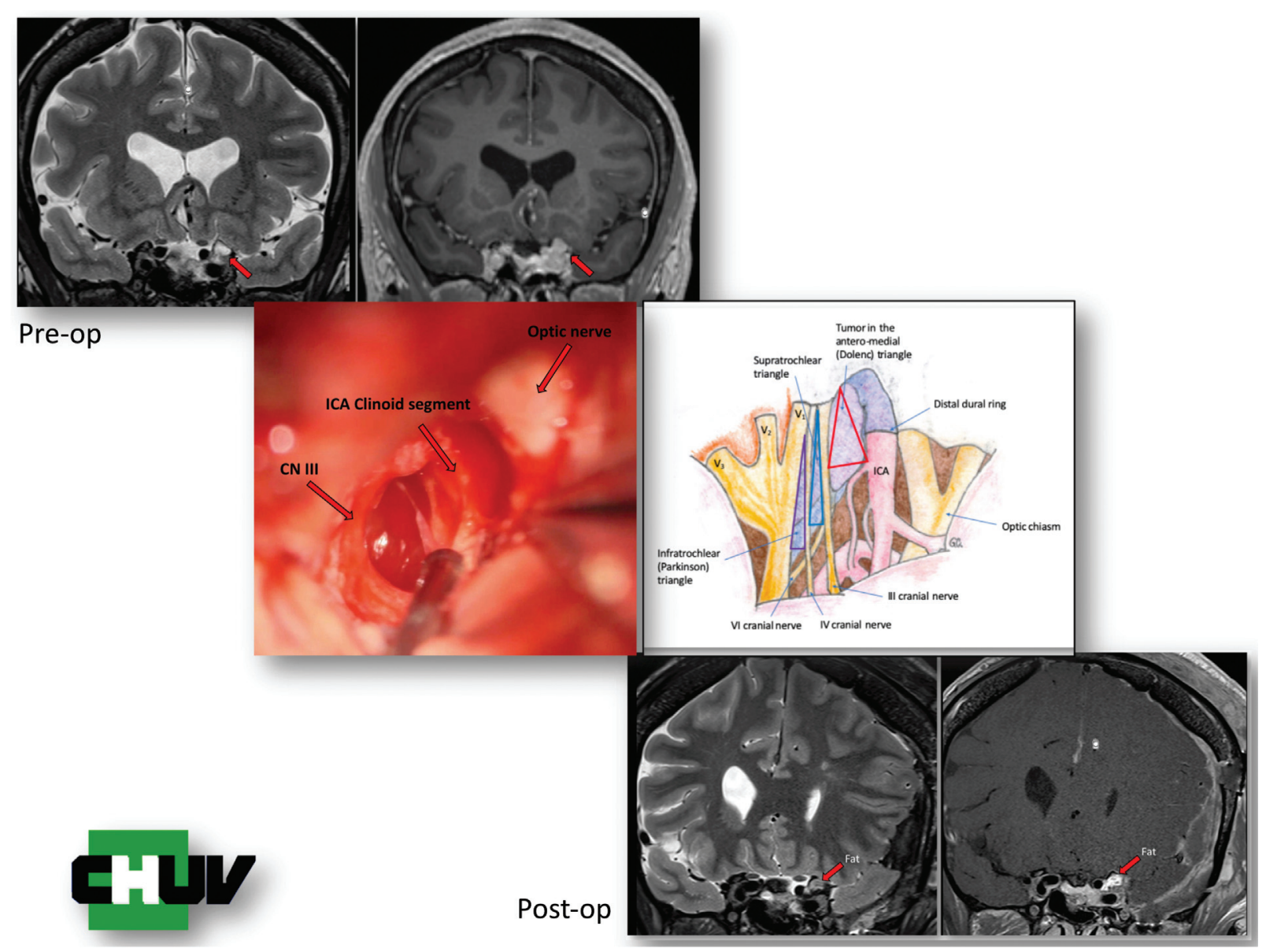

Fig. 1 Transcavernous approach to the anteromedial triangle (intraoperative picture and schematic drawing). Pre- and postoperative image.

Conflict of Interest

None declared. 